\title{
Fatores preditivos de hemotransfusão após artroplastia total de joelho*
}

\section{Predictive Factors for Blood Transfusion after Total Knee Arthroplasty}

\author{
Alan de Paula Mozella ${ }^{1}$ Hugo Alexandre de Araújo Barros Cobra ${ }^{10}$ Maria Eugênia Leite Duarte ${ }^{20}$ \\ ${ }^{1}$ Centro de Cirurgia do Joelho, Instituto Nacional de Traumatologia e \\ Ortopedia (INTO), Rio de Janeiro, RJ, Brasil \\ 2 Divisão de Pesquisa, Instituto Nacional de Traumatologia e \\ Ortopedia (INTO), Rio de Janeiro, RJ, Brasil \\ Endereço para correspondência Alan de Paula Mozella, M.D., M.Sc.,
Centro de Cirurgia do Joelho, Instituto Nacional de Traumatologia e \\ Ortopedia (INTO), Av. Brasil, 500, Caju, Rio de Janeiro, RJ, 20940-070, \\ Brasil (e-mail: apmozella@terra.com.br).
}

Rev Bras Ortop 2021;56(4):463-469.

\section{Resumo}

Palavras-chave

- artroplastia do joelho

- transfusão de sangue

- osteoartrite do joelho
Objetivos O presente estudo tem como objetivos (1) verificar a incidência e o volume de transfusão sanguínea entre os pacientes submetidos à artroplastia total do joelho (ATJ) unilateral cimentada em um único centro de referência nacional; (2) identificar variáveis pré e perioperatórias que nos permitam identificar os indivíduos sob maior risco (fatores preditores) quanto à necessidade de transfusão sanguínea nas 48 horas subsequentes à realização da cirurgia; (3) estimar o risco de transfusão sanguínea durante as primeiras 48 horas após o procedimento.

Métodos A amostra inicial foi constituída por todos os pacientes submetidos à AT] entre agosto de 2010 e agosto de 2013. Após aplicação dos critérios de exclusão, permaneceram no estudo 234 pacientes com idade entre 30 e 83 anos, portadores de osteaoartrose primária ou secundária a artrite reumatoide.

Resultados A análise dos resultados mostrou que valores de hemoglobina préoperatória $\leq 12,3 \mathrm{~g} / \mathrm{dL}$ e tempo de isquemia $\geq 87$ minutos são preditores independentes para hemotransfusão após AT], com risco relativo de 2,48 e 1,78, respectivamente. Aproximadamente metade dos pacientes $(51,3 \%)$ submetidos a ATJ com essas duas variáveis necessitaram de hemotransfusão.

Conclusão A incidência de transfusão sanguínea após ATJ foi de 33,7\%. Em média, cada paciente foi transfundido com $480 \mathrm{~mL}$ de concentrado de hemácias. Concentração de hemoglobina pré-operatória $\leq 12,3 \mathrm{~g} / \mathrm{dL}(p<0,001)$ e tempo de isquemia $\geq 87$ minutos $(p<0,047)$ foram preditores independentes para hemotransfusão em ATJ sob uso de manguito pneumático, com risco relativo de 2,48 e 1,78, respectivamente. A idade, o gênero, diagnóstico ou índice de massa corporal não
Trabalho desenvolvido no Centro de Cirurgia do Joelho do Instituto Nacional de Traumatologia e Ortopedia (INTO), Rio de Janeiro, RJ, Brasil. recebido 13 de Dezembro de 2019 aceito

01 de Junho de 2020

Publicado on-line

Novembro 2, 2020
DOI https://doi.org/ 10.1055/s-0040-1715511. ISSN 0102-3616. (c) 2020. Sociedade Brasileira de Ortopedia e Traumatologia. All rights reserved.

This is an open access article published by Thieme under the terms of the Creative Commons Attribution-NonDerivative-NonCommercial-License, permitting copying and reproduction so long as the original work is given appropriate credit. Contents may not be used for commercial purposes, or adapted, remixed, transformed or built upon. (https://creativecommons.org/ licenses/by-nc-nd/4.0/)

Thieme Revinter Publicações Ltda., Rua do Matoso 170, Rio de Janeiro, RJ, CEP 20270-135, Brazil 
foram considerados preditores independentes para a necessidade de hemotransfusão até 48 horas após o procedimento de artroplastia.

Abstract

\author{
Keywords \\ - arthroplasty, \\ replacement, knee \\ - blood transfusion \\ - osteoarthritis, knee
}

Objectives The present paper aims to (1) verify the incidence and volume of blood transfusion among patients undergoing unilateral cemented total knee arthroplasty (TKA) in a single Brazilian reference center; (2) identify pre- and perioperative variables to determine subjects with higher risk (i.e., predictive factors) for blood transfusion within 48 hours following surgery; (3) estimate the risk of blood transfusion during the first 48 hours after the procedure. Methods The initial sample consisted of all patients undergoing TKA from August 2010 to August 2013. After applying exclusion criteria, 234 patients aged 30 to 83 years old and diagnosed with primary or secondary osteoarthritis due to rheumatoid arthritis remained in the study.

Results Preoperative hemoglobin levels $\leq 12.3 \mathrm{~g} / \mathrm{dL}$ and ischemia time $\geq 87$ minutes were independent predictors for post-TKA blood transfusion, with a relative risk of 2.48 and 1.78 , respectively. Approximately half of the TKA patients (51.3\%) presenting these two variables required a blood transfusion.

Conclusion The incidence of post-TKA blood transfusion was 33.7\%. On average, each transfused patient received $480 \mathrm{~mL}$ of packed red blood cells. Preoperative hemoglobin levels $\leq 12.3 \mathrm{~g} / \mathrm{dL}(p<0.001)$ and ischemia time $\geq 87$ minutes $(p<0.047)$ were independent predictors for blood transfusion in TKA using a pneumatic cuff, with a relative risk of 2.48 and 1.78, respectively. Age, gender, diagnosis, or body mass index were not considered independent predictors for the need for blood transfusion up to 48 hours after the procedure.

\section{Introdução}

A artroplastia total do joelho (ATJ) normalmente se associa à expressiva perda sanguínea visível, podendo ultrapassar $1.500 \mathrm{~mL} .^{1}$ Tais perdas volêmicas são, ainda, subestimadas por desconsiderar o extravasamento de sangue para tecidos moles traumatizados, formação de hematoma na cavidade articular, e hemólise. Cerca de $80 \%$ desse sangramento oculto ocorre nas primeiras 24 horas, podendo corresponder até $50 \%$ da perda sanguínea total real. ${ }^{1-3}$

Perdas volêmicas desta magnitude frequentemente resultam em necessidade de transfusão sanguínea em 7,1 a 67\% dos pacientes. ${ }^{4-9}$ Embora as complicações relacionadas à hemotransfusão tenham diminuído nas últimas décadas, os riscos não são nulos. ${ }^{8-12}$ A transfusão sanguínea alogênica também é associada à elevação do risco de infecção após cirurgias com implantes ortopédicos. Tais evidências, contudo, permanecem inconclusivas e carecem de estudos futuros. ${ }^{10-17}$

Desse modo, a possibilidade de identificar, no período préoperatório, quais os pacientes sob maior risco de receberem transfusão sanguínea no pós-operatório de ATJ pode auxiliar a mudança de medidas nos períodos pré, intra e pós-peratórios. ${ }^{7}$

Assim, os objetivos do presente trabalho são: (1) verificar a incidência e o volume de transfusão sanguínea entre os pacientes submetidos à ATJ unilateral cimentada em um único centro; (2) identificar fatores preditivos quanto à necessidade de transfusão sanguínea nas 48 horas subsequentes à ATJ; (3) estimar o risco de transfusão sanguínea durante as primeiras 48 horas após o procedimento.

\section{Material e Métodos}

Após aprovação pelo Comitê de Ética em Pesquisa da Instituição, realizamos avaliação retrospectiva dos prontuários de todos os pacientes submetidos à artroplastia total unilateral cimentada de joelho por dois cirurgiões, no período entre agosto de 2010 e agosto de 2013.

Foram excluídos da amostra os pacientes submetidos à artroplastia unicompartimental do joelho (AUJ), conversão de AUJ para ATJ, cirurgia bilateral, revisão de ATJ, portadores de coagulopatias ou em terapia anticoagulante pré-operatória. Nenhum paciente realizou doação de sangue autólogo pré-operatório.

Foram incluídos no estudo pacientes de ambos os gêneros, com idade entre 30 e 83 anos, portadores de osteoartrose (OA) primária ou de artrite reumatóide. Todos os procedimentos foram realizados por acesso parapatelar medial após anestesia raquidiana associada a bloqueio periférico do nervo isquiático e do nervo femoral. As cirurgias foram realizadas sob isquemia com pressão de $300 \mathrm{~mm} \mathrm{Hg}$, aplicada antes da incisão. Após cimentação dos implantes do sistema P. F. C. Sigma (DePuySynthes Companies, Warsaw, IN, EUA) póstero-estabilizado e com substituição patelar, a isquemia foi liberada para a realização de hemostasia apenas com eletrocautério monopolar. Não foram administrados fármacos de ação hemostática. Em todos os pacientes foi colocado dreno único de $4,8 \mathrm{~mm}$, mantido por 24 horas. A profilaxia antimicrobiana foi feita com cefazolina ( $2 \mathrm{~g}$ endovenosa durante a indução anestésica), mantida por 24 horas ( $1 \mathrm{~g}$ endovenosa $8 / 8$ horas). A prevenção 
de trombose venosa profunda (TVP) foi realizada com heparina de baixo peso molecular por via subcutânea (Clexane SanofiAvenitis, São Paulo, SP, Brasil) em dose única diária de $40 \mathrm{mg}$, iniciada 12 horas após a cirurgia e mantida por 10 dias.

As variáveis pré-operatórias pesquisadas foram: gênero, idade, diagnóstico, índice de massa corporal (IMC) segundo critérios da Organização Mundial de Saúde (OMS) do ano 2000, risco cirúrgico segundo critérios da Sociedade Americana de Anestesiologia (ASA) e concentração de hemoglobina $(\mathrm{Hb})$ aferida 24 horas antes do procedimento cirúrgico. 0 tempo de isquemia foi o parâmetro intraoperatório avaliado. No pós-operatório imediato, determinado como as 48 horas subsequentes à cirurgia, foram registradas a menor concentração de $\mathrm{Hb}$ do período e o volume total de hemoconcentrados alogênicos transfundidos.

A necessidade de hemotransfusão alogênica durante a cirurgia foi determinada pelo anestesiologista que não desempenhou função no estudo. A hemotransfusão no pós-operatório foi determinada pela equipe assistente observando protocolo de hemotransfusão adotado pela instituição, em que os pacientes com $\mathrm{Hb}$ superior a $10 \mathrm{~g} / \mathrm{dL}$ não necessitam de transfusão sanguínea. Para os pacientes com níveis de $\mathrm{Hb}$ entre 6 e $7 \mathrm{~g} / \mathrm{dL}$ é indicada a realização de hemotransfusão. Para valores de $\mathrm{Hb}$ entre 7 e $10 \mathrm{~g} / \mathrm{dL}$, a decisão é tomada considerando-se a situação individualmente. Após o término desse período, os pacientes foram divididos em dois grupos, segundo a necessidade de transfusão de hemoconcentrados: transfundidos (grupo T, $n=79$ ) e não transfundidos (grupo nT, $n=155$ ).

\section{Análise Estatística}

O teste de normalidade de Kolmogorov-Smirnov (e ShapiroWilks) foi aplicado para verificar se a variável apresentava distribuição normal.

A existência de relação significativa entre as variáveis estudadas com a necessidade de transfusão sanguínea após a ATJ foi avaliada pelos seguintes métodos: (1) teste $t$ de Student para comparação de dados numéricos e MannWhitney para amostras independentes; (2) análise inferencial pelo coeficiente de correlação de Spearman para medir o grau de associação entre variação da hemoglobina $(\Delta \mathrm{Hb})$, tempo de isquemia e volume transfundido; (3) teste de $X^{2}$ ou exato de Fisher para comparação de dados categóricos; (4) análise de regressão logística para avaliar a influência simultânea das variáveis preditoras. 0 processo de seleção das variáveis foi o de stepwise forward, ao nível de $5 \%$.

A curva característica de operação do receptor (COR) foi construída para determinar a acurácia do modelo em predizer a necessidade de hemotransfusão. Essa representação gráfica é obtida pela fração sensibilidade/especificidade em vários pontos de corte de cada variável e, deste modo, ilustra o desempenho de um sistema e seu limiar de discriminação. Pode-se também identificar o melhor ponto de corte (cut-off).

Foram consideradas diferenças estatisticamente significativas para valores de $p<0,05$. Todos os cálculos foram realizados por estatístico independente, utilizando software estatístico SAS versão 6.11 (SAS Institute, Inc., Cary, NC, EUA).

\section{Resultados}

Incidência e Volume de Transfusão Sanguínea Alogênica

A amostra inicial era formada por 237 pacientes. Após exclusão de 3 pacientes por serem portadores de hemofilia, permaneceram no estudo 234 pacientes com idade média de $66,6 \pm 9,1$ anos (faixa entre 30-83 anos). Deste total, $81,6 \%$ (191/234) eram mulheres. Quanto ao diagnóstico, 83,8\% (196/234) eram portadores de OA idiopática, e 16,2\% (38/234) foram diagnosticados com AR.

A incidência de transfusão sanguínea após ATJ foi de 33,7\% (79/234). Em média, cada paciente foi transfundido com $480 \mathrm{~mL}$ (1,6 unidades) de concentrado de hemácias. Cerca da metade $(50,6 \%)$ dos pacientes que foram transfundidos, recebeu um volume total de $600 \mathrm{~mL}$ de hemoconcentrados, $35(44,3 \%)$ pacientes necessitaram de $300 \mathrm{~mL}$, sendo que apenas 5,1\% (4/79) dos pacientes necessitaram de mais 2 unidades de hemocomponentes.

$\mathrm{Na}$-Tabela 1 estão resumidas as características gerais dos pacientes alocados no grupo $\mathrm{T}$ (transfundido), e grupo $\mathrm{nT}$ (não transfundido). Não houve diferença entre os grupos estudados quanto à idade, gênero, diagnóstico e índice de massa corporal e ASA ( - Tabela $\mathbf{1}$ ).

\section{Fatores Preditores da Necessidade de Transfusão Sanguínea Alogênica}

A análise univariada dos parâmetros clínicos e cirúrgicos demonstrou que a necessidade de transfusão sanguínea após ATJ está relacionada com baixa concentração de Hb no pré e

Tabela 1 Características e variáveis numéricas clínicas dos pacientes $(n=234)$

\begin{tabular}{|c|c|c|c|}
\hline & $\begin{array}{l}\text { Grupo T } \\
(n=79)\end{array}$ & $\begin{array}{l}\text { Grupo } n T \\
(n=155)\end{array}$ & $\begin{array}{l}\text { Valor } \\
\text { de } p\end{array}$ \\
\hline Idade* & $67,6 \pm 9,3$ & $66,1 \pm 9,0$ & 0,23 \\
\hline \multicolumn{4}{|l|}{ Gênero** $^{* *}$} \\
\hline$M$ & $10(12,7 \%)$ & $33(21,3 \%)$ & \multirow[t]{2}{*}{0,10} \\
\hline $\mathrm{F}$ & $69(87,3 \%)$ & $122(78,7 \%)$ & \\
\hline \multicolumn{4}{|l|}{ Diagnóstico ${ }^{* *}$} \\
\hline$A R$ & $10(12,7 \%)$ & $28(18,5 \%)$ & \multirow[t]{2}{*}{0,25} \\
\hline $\mathrm{OA}$ & $69(87,3 \%)$ & $123(81,5 \%)$ & \\
\hline IMC $\left(\mathrm{kg} / \mathrm{m}^{2}\right)^{*}$ & $30 \pm 5,4$ & $30,2 \pm 5,3$ & 0,71 \\
\hline \multicolumn{4}{|l|}{$\mathrm{ASA}^{* *}$} \\
\hline 1 & 3 & 11 & \multirow[t]{3}{*}{0,49} \\
\hline 2 & 75 & 139 & \\
\hline 3 & 1 & 5 & \\
\hline
\end{tabular}

Abbreviations: AR, artrite reumatóide; ASA, American Society Anestesiology; F, feminino; IMC, índice de massa corporal; M, masculino; OA, osteoartrose.

Dados numéricos expressos pela média \pm desvio padrão. Dados categóricos expressos em frequência e percentual.

Grupo T= recebeu hemotransfusão pós-operatória, Grupo nT= não recebeu hemotransfusão pós-operatória.

*teste $t$ de Student para amostras independentes ou de Mann-Whitney. "*teste de $\mathrm{X} 2$ ou exato de Fisher. 
Tabela 2 Análise univariada dos parâmetros numéricos $(n=234)$

\begin{tabular}{|l|l|l|l|l|l|}
\hline Variável & \multicolumn{3}{|l|}{ Grupo T $(\boldsymbol{n}=\mathbf{7 9})$} & Grupo $\mathrm{nT}(\boldsymbol{n}=\mathbf{1 5 5})$ & \multicolumn{2}{l|}{ Valor de $\boldsymbol{p}^{\text {a }}$} \\
\hline Idade $($ anos) & $67,6 \pm 9,3$ & $(40-83)$ & $66,1 \pm 9,0$ & $(30-82)$ & 0,23 \\
\hline IMC $\left(\mathrm{Kg} / \mathrm{m}^{2}\right)$ & $30,0 \pm 5,4$ & $(20,8-44,9)$ & $30,2 \pm 5,3$ & $(15,0-43,8)$ & 0,71 \\
\hline Tempo de isquemia $(\mathrm{min})$ & $94,5 \pm 21,0$ & $(50-150)$ & $87,0 \pm 20,2$ & $(40-135)$ & 0,0014 \\
\hline
\end{tabular}

Abreviatura: IMC, índice de massa corporal.

Grupo T= recebeu hemotransfusão pós-operatória, Grupo nT= não recebeu hemotransfusão pós-operatória. Dados expressos pela média \pm desvio padrão com amplitude entre parênteses.

ateste $t$ de Student para amostras independentes.

Tabela 3 Análise univariada dos parâmetros categóricos

\begin{tabular}{|c|c|c|c|c|c|}
\hline Variável & \multicolumn{2}{|c|}{$\begin{array}{l}\text { Grupo T } \\
(n=79)\end{array}$} & \multicolumn{2}{|c|}{$\begin{array}{l}\text { Grupo nT } \\
(n=155)\end{array}$} & Valor de $p^{a}$ \\
\hline \multicolumn{6}{|l|}{ Sexo } \\
\hline$M$ & 10 & $12,7 \%$ & 33 & $21,3 \%$ & \multirow[t]{2}{*}{0,10} \\
\hline $\mathrm{F}$ & 69 & 87,3 & 122 & 78,7 & \\
\hline \multicolumn{6}{|c|}{ Diagnóstico } \\
\hline $\mathrm{OA}$ & 69 & 87,3 & 122 & 78,7 & \multirow[t]{2}{*}{0,25} \\
\hline AR & 10 & 12,7 & 33 & 21,3 & \\
\hline \multicolumn{6}{|c|}{ Risco cirúrgico } \\
\hline ASA 1 & 3 & 3,9 & 11 & 7,1 & \multirow[t]{3}{*}{0,49} \\
\hline ASA 2 & 74 & 94,9 & 139 & 89,7 & \\
\hline ASA 3 & 1 & 1,3 & 5 & 3,2 & \\
\hline
\end{tabular}

Abbreviations: AR, artrite reumatóide; OA, osteoartrose.

Grupo $\mathrm{T}=$ recebeu hemotransfusão pós-operatória, Grupo nT= não recebeu hemotransfusão pós-operatória.

ateste $x^{2}$ ou exato de Fisher.

no pós-operatório. Observamos também que os pacientes que receberam transfusão apresentaram maior tempo de isquemia $(p<0,0014)$. As demais variáveis, como diagnós- tico, gênero, idade, IMC e risco cirúrgico, não apresentaram relação com a necessidade de hemotransfusão pós-operatória (-Tabelas 2 e 3 ).

Em ambos os gêneros, observou-se que o grupo T apresentou concentrações de Hb no pré-operatório e no pósoperatório significativamente menores do que o grupo nT. De modo semelhante, a variação dos níveis de $\mathrm{Hb}(\Delta \mathrm{Hb}$ absolutos e relativos) foi significativamente maior em relação ao grupo nT em ambos os gêneros (-Tabela 4 ).

Nos pacientes que receberam a hemotransfusão (grupo T), quando analisada a relação entre a variação de $\mathrm{Hb}(\Delta \mathrm{Hb})$ pré e pós-operatória e o tempo de isquemia, verificamos a existência de correlação significativa entre o $\Delta \mathrm{Hb}$ absoluto $\left(\mathrm{r}_{\mathrm{s}}=0,18 ; p=0,006\right)$ e relativo $\left(\mathrm{r}_{\mathrm{s}}=0,17 ; p=0,007\right)$ com o tempo de isquemia.

Quando analisada a relação entre a $\Delta \mathrm{Hb}$ pré e pós-operatória com o volume de hemoderivados transfundidos, verificamos a existência de correlação significativa entre a $\Delta \mathrm{Hb}$ absoluta $\left(\mathrm{r}_{\mathrm{s}}=0,24 ; p=0,0002\right)$ e relativa $\left(\mathrm{r}_{\mathrm{s}}=0,35 ; p=0,0001\right)$ com o volume final transfundido. Foi observada também correlação significativa $\left(r_{s}=0,16 ; p=0,013\right)$ entre o tempo de isquemia e o volume de hemoderivados transfundido para os pacientes.

O tempo de isquemia também mostra relação com uma maior queda absoluta e relativa nos valores da $\mathrm{Hb}$. A queda

Tabela 4 Concentração de hemoglobina $(\mathrm{Hb})$ e variações $(\Delta)$ segundo transfusão estratificado por gênero

\begin{tabular}{|c|c|c|c|c|c|}
\hline Variável & \multicolumn{2}{|l|}{ Grupo T } & \multicolumn{2}{|l|}{ Grupo nT } & Valor de $p^{a}$ \\
\hline \multicolumn{6}{|l|}{ Gênero masculino $n=10 n=33$} \\
\hline Hb no pré-operatório (g/dL) & $12,9 \pm 0,7$ & $(11,8-14,1)$ & $13,8 \pm 1,4$ & $(11,2-16,7)$ & 0,046 \\
\hline Hb no pós-operatório (g/dL) & $8,0 \pm 1,1$ & $(6,7-10)$ & $9,9 \pm 1,5$ & $(7,3-14,2)$ & 0,0007 \\
\hline$\Delta \mathrm{Hb}(\mathrm{g} / \mathrm{dL})^{1}$ & $5,0 \pm 1,1$ & $(6,7-3)$ & $4,0 \pm 1,3$ & $(6,7-1,1)$ & 0,033 \\
\hline$\Delta \mathrm{Hb}(\%)^{2}$ & $38,3 \pm 8,0$ & $(50-24)$ & $28,7 \pm 8,7$ & $(48-9,4)$ & 0,003 \\
\hline Gênero feminino & \multicolumn{2}{|l|}{$\mathrm{n}=69$} & \multicolumn{2}{|l|}{$\mathrm{n}=122$} & \\
\hline Hb no pré-operatório (g/dL) & $11,9 \pm 1,3$ & $(9,3-14,8)$ & $12,4 \pm 1,2$ & $(8,7-16,1)$ & 0,002 \\
\hline Hb no pós-operatório (g/dL) & $7,8 \pm 1,0$ & $(5,8-10,9)$ & $9,1 \pm 1,4$ & $(6,1-12,3)$ & 0,0001 \\
\hline$\Delta \mathrm{Hb}(\mathrm{g} / \mathrm{dL})^{1}$ & $4,1 \pm 1,6$ & $(7,4-1,3)$ & $3,3 \pm 1,4$ & $(8,2-1,1)$ & 0,0005 \\
\hline$\Delta \mathrm{Hb}(\%)^{2}$ & $34,0 \pm 10,5$ & $(54-12,7)$ & $26,5 \pm 9,8$ & $(51-9,1)$ & 0,0001 \\
\hline
\end{tabular}

Abreviatura: Hb, hemoglobina.

Grupo T = recebeu hemotransfusão pós-operatória, Grupo nT= não recebeu hemotransfusão pós-operatória. Dados expressos pela média \pm desvio padrão com amplitude entre parênteses.

${ }^{1} \Delta$ absoluto $=\mathrm{Hb}$ pré $-\mathrm{Hb}$ pós.

${ }^{2} \Delta$ relativo $(\%)=\Delta$ absoluto/Hb pré ${ }^{*} 100$.

ateste $t$ de Student para amostras independentes. 

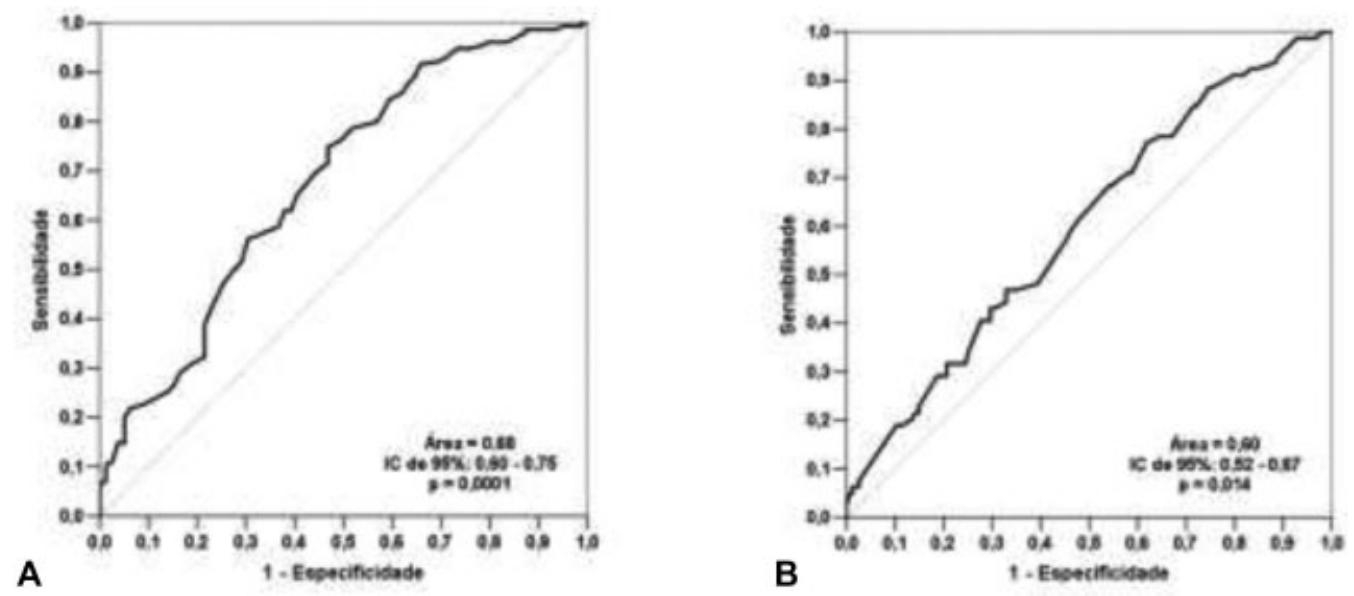

Fig. 1 Acurácia global medida pela curva característica de operação do receptor da hemoglobina pré-operatória (A) e do tempo de isquemia (B) para hemotransfusão após artroplastia total do joelho sob isquemia com manguito pneumático.

dos níveis de $\mathrm{Hb}$ e o tempo de isquemia, por sua vez, estão diretamente relacionados com maior volume de hemoderivados transfundido no peroperatório de ATJ.

Uma subsequente análise de regressão logística multivariada foi realizada para avaliar a influência simultânea de variáveis preditoras sobre a necessidade de hemotransfusão. Essa avaliação confirmou a concentração pré-operatória de $\mathrm{Hb}(p<0,0001)$ e tempo de isquemia como fatores preditores independentes quanto a necessidade de hemotransfusão pós-operatória. As demais variáveis não apresentaram contribuição independente significativa, ao nível de $5 \%$.

Realizamos a construção da curva COR para $\mathrm{Hb}$ e para tempo de isquemia, identificando o cut-off para hemotransfusão após ATJ nos pacientes com concentração pré-operatória de $\mathrm{Hb} \leq 12,3 \mathrm{~g} / \mathrm{dL}$. A sensibilidade desse teste foi de $62,0 \%$ e a especificidade de $61,9 \%$. Na curva do tempo de isquemia, identificamos o cut-off nos pacientes com tempo de isquemia $\geq 87$ minutos, com sensibilidade de $62,0 \% \mathrm{e}$ especificidade de $51,6 \%$ ( - Figura $\mathbf{1}$ ).

Ao realizar regressão logística após identificação dos cutoff, observamos que valores de $\mathrm{Hb}$ pré-operatórios $\leq 12,3$ $\mathrm{g} / \mathrm{dL} \quad(p<0,001)$ e tempo de isquemia $\geq 87$ minutos $(p<0,047)$ foram preditores independentes para hemotransfusão em ATJ sob uso de manguito pneumático, com risco relativo de 2,48 e 1,78 , respectivamente.

Tabela 5 Probabilidade estimada de hemotransfusão segundo modelo logístico

\begin{tabular}{|l|l|l|l|}
\hline $\begin{array}{l}\text { Hb } \\
\text { pré-operatória } \\
\mathbf{1 2 , 3} \mathbf{g} / \mathrm{dL}\end{array}$ & $\begin{array}{l}\text { Tempo de } \\
\text { isquemia } \\
\mathbf{8 7 7} \text { minutes }\end{array}$ & $\begin{array}{l}\text { Probabilidade } \\
\text { estimada (\%) }\end{array}$ & $\begin{array}{l}\text { Intervalo de } \\
\text { confiança } \\
\text { de } \mathbf{9 5 \%}\end{array}$ \\
\hline Não & Não & 19,3 & $12,4-28,9$ \\
\hline Não & Sim & 29,9 & $20,8-40,8$ \\
\hline Sim & Não & 37,2 & $26,4-49,4$ \\
\hline $\operatorname{Sim}$ & Sim & 51,3 & $40,0-62,6$ \\
\hline
\end{tabular}

Abreviatura: $\mathrm{Hb}$, hemoglobina.

\section{Risco de transfusão sanguínea alogênica após ATJ}

Com base no modelo da regressão logística, a probabilidade do paciente submetido à ATJ necessitar de transfusão sanguínea nas 48 horas subsequentes ao procedimento pode ser calculada pela seguinte fórmula:

$$
\left.\operatorname{Pr}_{\text {est }} \text { (hemotransfusão }\right)=\frac{\mathrm{e}^{3,7596-(0,5178 \times \mathrm{Hb} \text { pré em g/dL })+}}{(0,0211 \times \text { tempo de cirurgia min })}
$$

Assim, a probabilidade estimada de transfusão de acordo com os parâmetros relevantes analisados é apresentada na - Tabela 5.

\section{Discussão}

O presente estudo é uma avaliação transversal de 234 pacientes submetidos à ATJ que demonstra que concentração pré-operatória de $\mathrm{Hb} \leq 12,3 \mathrm{~g} / \mathrm{dL}$ e tempo de isquemia $\geq$ 87 minutos são preditores independentes para hemotransfusão após ATJ.

Apesar de as cirurgias de ATJ normalmente se associarem à elevada perda sanguínea total, ampla variação nas taxas de hemotransfusão é observada na literatura. Em nossa amostra, a transfusão foi realizada em 33,7\% dos pacientes dentro de 48 horas após a cirurgia. Apesar de este resultado ser semelhante ao de outros autores descritos em uma grande série de pacientes, ${ }^{9}$ é possível que a adoção de critérios institucionais conservadores para indicação de transfusão sanguínea possa acarretar na redução dessa taxa. Ballantyne et al. ${ }^{17}$ identificaram redução da incidência de hemotransfusão pós-ATJ unilateral de 31 para 11,9\% após adoção de protocolo transfusional institucional mais rigoroso, indicando hemotransfusão apenas em pacientes que apresentassem concentração de $\mathrm{Hb}<8,5 \mathrm{~g} / \mathrm{dL}$ no pós-operatório ou na presença de sintomas de anemia aguda.

Modelos preditores que possibilitem identificar pacientes com maior probabilidade de necessidade de hemotransfusão pós-operatória são essenciais para a gestão racional de 
hemocomponentes. ${ }^{7}$ Assim, buscamos avaliar na população brasileira variáveis pré e perioperatórias que nos permitissem identificar os indivíduos sob maior risco de necessitar de hemotransfusão nas 48 horas subsequentes à ATJ.

$O$ preditor independente mais consistente da necessidade de transfusão pós-operatória foi a concentração pré-operatória de $\mathrm{Hb} \leq 12,3 \mathrm{~g} / \mathrm{dL}$. A partir desse valor, o risco relativo de transfusão foi de 2,48 , sugerindo, portanto, que pacientes com essa taxa de $\mathrm{Hb}$ têm 2,48 vezes mais chances de necessitarem de hemotransfusão quando submetidos à ATJ do que pacientes com a taxa acima desse valor. Salido et al. ${ }^{18}$ também identificaram a concentração pré-operatória de $\mathrm{Hb}<13 \mathrm{~g} / \mathrm{dL}$ como fator preditor de hemotransfusão em pacientes submetidos à artroplastia total do quadril (ATQ) ou ATJ, com um risco relativo semelhante ao encontrado no nosso grupo analisado (2,51). 0 percentual de pacientes dentro desta faixa de valores de $\mathrm{Hb}$ pré-operatória que foram transfundidos (69\%) também foi semelhante ao observado na nossa casuística (62\%). Resultados semelhantes foram observados por Guerin et al. ${ }^{19}$ ao identificar risco 4 vezes maior de transfusão nos pacientes com concentração pré-operatória de $\mathrm{Hb}<13 \mathrm{~g} / \mathrm{dL}$, risco este potencializado em pacientes ASA 3 e $4 .^{9}$

Outro parâmetro preditor independente para hemotransfusão observado neste estudo foi o tempo de isquemia $\geq$ 87 minutos, acarretando num risco relativo de 1,78 . Os resultados de Noticewala et al. ${ }^{20}$ mostraram que apenas o tempo cirúrgico total (e não o tempo de isquemia) é um fator preditivo independente da necessidade de hemotransfusão pós-ATJ. Embora Salido et al. ${ }^{18}$ tenham relatado que o tempo cirúrgico de 91,1 minutos estava associado à maior necessidade de hemotransfusão, este achado não foi confirmado como fator preditor independente para hemotransfusão.

Prasad et al. $^{3}$ confirmaram haver maior necessidade de transfusão sanguínea após ATJ em portadores de AR. Entretanto, Ogbemudia et al. ${ }^{21}$ identificaram a baixa concentração de $\mathrm{Hb}$ como sendo fator preditor para transfusão em pacientes com AR. No nosso estudo, à semelhança de Noticewala et al., 20 o diagnóstico de AR não foi um fator preditor independente. Contudo, como também observado por Ogbemudia et al., ${ }^{21} \mathrm{a}$ baixa concentração de $\mathrm{Hb}$ nesses pacientes foi um fator de risco para transfusão sanguínea pós-operatória.

A idade foi identificada por Hart et al. ${ }^{6}$ como sendo fator preditor independente, com elevação de 10,2 vezes do risco de transfusão para cada década de vida. Nossos resultados não confirmaram a relação entre idade e necessidade de transfusão após ATJ.

À semelhança da idade, o gênero do paciente não foi um preditor independente de transfusão pós-ATJ. Apesar das mulheres, em geral, apresentarem menores concentrações de $\mathrm{Hb}$, no nosso estudo, assim como no de Ogbemudia et al. ${ }^{21}$ não houve relação entre o gênero do paciente e a necessidade de transfusão.

O IMC é outro parâmetro frequentemente investigado. Menores valores de IMC são descritos como estando associados à menor ${ }^{13,14}$ ou a maior ${ }^{22}$ necessidade transfusional. O peso corporal foi descrito por Salido et al. ${ }^{18}$ como sendo um fator preditor independente para a necessidade de hemotransfusão pós-operatória, com risco relativo de 1,05. Em portadores de AR, o IMC mostrou relação com maior necessidade de hemotransfusão. ${ }^{21}$ No presente estudo, assim como no de Bong et al., ${ }^{23}$ não foi observada a relação entre o IMC e a necessidade de transfusão pós-operatória.

A literatura aponta para um consenso de que pacientes com ASA superior a 2, em geral, necessitam de transfusão, 6,7,9,11,15 demostrando que, em relação aos pacientes ASA 1 e 2, os pacientes ASA 3 e 4 têm um risco independente para a necessidade de hemotransfusão pós-operatória 3 vezes maior. Na nossa amostra, não observamos relação entre risco cirúrgico (ASA) e a necessidade de hemotransfusão, o que pode ser explicado pelo número reduzido de participantes do estudo com ASA igual ou maior do que 3 .

A identificação, no período pré-operatório, dos pacientes sob maior risco de necessitar de transfusão sanguínea no pós-operatório de ATJ, tem uma importância central no cenário da saúde pública brasileira. Assim, desenvolvemos um modelo que permite estimar o risco relativo do paciente necessitar de hemotransfusão sanguínea. A curva COR foi construída para determinar a acurácia desse modelo em predizer quais pacientes apresentavam risco transfusional elevado após ATJ. O poder discriminatório de nosso modelo, estimado pela curva COR da Hb (área sob a curva de 0,68), embora regular, atingiu significância estatística com sensibilidade de $62,0 \%$ e especificidade de $61,9 \%$. Quando avaliada a curva COR do tempo de isquemia (área sob a curva de $0,60)$, o poder preditivo de nossa equação foi baixo, mas com relevância estatística e com sensibilidade de $62,0 \%$ e especificidade de $51,6 \%$. Outros modelos preditivos realizados em condições semelhantes às do nosso estudo mostraram maior poder discriminatório do que a nossa equação, com área sob a curva de $0,74^{22}$ ou $0,99^{24}$ e sensibilidade de $71 \%{ }^{22}$ ou $90 \%{ }^{18}$ Contudo, a especificidade deste último relato $(52,5 \%)$ foi semelhante à encontrada no nosso estudo $(51,6 \%){ }^{20}$

Acreditamos, entretanto, que antes da utilização da equação nas tomadas de decisão, seja importante a sua validação em outras instituições para possíveis adequações do modo.

Nosso estudo apresenta algumas limitações. Apesar da existência de parâmetros clínicos e laboratoriais a serem observados no protocolo transfusional, a visão do especialista (anestesiologista, intensivista, clínicos e cirurgiões) pode ter particularizado a indicação transfusional em alguns pacientes, o que configura limitação desse estudo. Outra importante limitação refere-se a não realização do cálculo amostral; entretanto, acreditamos que o fato da nossa amostra ser constituída por um número expressivo de pacientes, originados de um único centro terciário de referência no tratamento de doenças ortopédicas de alta complexidade, imputa aos resultados importante relevância. Destacamos, porém, a necessidade de estudos adicionais prospectivos para corroborar nossos resultados e para orientar o tratamento mais adequado dos pacientes em programação pré-operatória de ATJ que apresentem concentração de $\mathrm{Hb}<12,3 \mathrm{~g} / \mathrm{dL}$. Do mesmo modo, verificamos a necessidade de adoção de medidas efetivas visando a redução de sangramento em pacientes nos quais as cirurgias transcorram com tempo de isquemia $>87$ minutos. 


\section{Conclusão}

A incidência de transfusão sanguínea após ATJ foi de 33,7\%. Em média, cada paciente foi transfundido com $480 \mathrm{~mL}$ de concentrado de hemácias.

Concentração de $\mathrm{Hb}$ pré-operatória $\leq 12,3 \mathrm{~g} / \mathrm{dL}$ $(p<0,001)$ e tempo de isquemia $\geq 87$ minutos $(p<0,047)$ foram preditores independentes para hemotransfusão em ATJ sob uso de manguito pneumático, com risco relativo de 2,48 e 1,78 , respectivamente.

A idade, o gênero, diagnóstico ou IMC não foram considerados preditores independentes para a necessidade de hemotransfusão até 48 horas pós-ATJ.

\section{Suporte Financeiro}

Não houve suporte financeiro de fontes públicas, comerciais, ou sem fins lucrativos.

\section{Conflito de Interesses}

Os autores declaram não haver conflito de interesses.

\section{Agradecimentos}

Agradecemos aos colegas ortopedistas Diego Perez da Motta e Daniel Ramallo pela colaboração na assistência aos pacientes.

\section{Referências}

1 Sehat KR, Evans RL, Newman JH. Hidden blood loss following hip and knee arthroplasty. Correct management of blood loss should take hidden loss into account. J Bone Joint Surg Br 2004;86(04): 561-565

2 Marulanda GA, Ragland PS, Seyler TM, Mont MA. Reductions in blood loss with use of a bipolar sealer for hemostasis in primary total knee arthroplasty. Surg Technol Int 2005;14:281-286

3 Prasad N, Padmanabhan V, Mullaji A. Blood loss in total knee arthroplasty: an analysis of risk factors. Int Orthop 2007;31(01): 39-44

4 Pedersen AB, Mehnert F, Overgaard S, Johnsen SP. Allogeneic blood transfusion and prognosis following total hip replacement: a population-based follow up study. BMC Musculoskelet Disord 2009; $10: 167$

5 Browne JA, Adib F, Brown TE, Novicoff WM. Transfusion rates are increasing following total hip arthroplasty: risk factors and outcomes. J Arthroplasty 2013;28(8, Suppl)34-37

6 Hart A, Khalil JA, Carli A, Huk O, Zukor D, Antoniou J. Blood transfusion in primary total hip and knee arthroplasty. Incidence, risk factors, and thirty-day complication rates. J Bone Joint Surg Am 2014;96(23):1945-1951

7 Levine BR, Haughom B, Strong B, Hellman M, Frank RM. Blood management strategies for total knee arthroplasty. J Am Acad Orthop Surg 2014;22(06):361-371

8 Rosencher N, Kerkkamp HE, Macheras G, et al. OSTHEO Investigation. Orthopedic Surgery Transfusion Hemoglobin European Over- view (OSTHEO) study: blood management in elective knee and hip arthroplasty in Europe. Transfusion 2003;43(04):459-469

9 Basora M, Tió M, Martin N, et al. Should all patients be optimized to the same preoperative hemoglobin level to avoid transfusion in primary knee arthroplasty? Vox Sang 2014;107(02):148-152

10 Frisch NB, Wessell NM, Charters MA, Yu S, Jeffries JJ, Silverton CD. Predictors and complications of blood transfusion in total hip and knee arthroplasty. J Arthroplasty 2014;29(9, Suppl)189-192

11 Klein HG. How safe is blood, really? Biologicals 2010;38(01): 100-104

12 Barsoum WK, Klika AK, Murray TG, Higuera C, Lee HH, Krebs VE. Prospective randomized evaluation of the need for blood transfusion during primary total hip arthroplasty with use of a bipolar sealer. J Bone Joint Surg Am 2011;93(06):513-518

13 Friedman R, Homering M, Holberg G, Berkowitz SD. Allogeneic blood transfusions and postoperative infections after total hip or knee arthroplasty. J Bone Joint Surg Am 2014;96(04): 272-278

14 Newman ET, Watters TS, Lewis JS, et al. Impact of perioperative allogeneic and autologous blood transfusion on acute wound infection following total knee and total hip arthroplasty. J Bone Joint Surg Am 2014;96(04):279-284

15 Rohde JM, Dimcheff DE, Blumberg N, et al. Health care-associated infection after red blood cell transfusion: a systematic review and meta-analysis. JAMA 2014;311(13):1317-1326

16 Gómez-Lesmes SP, Tornero E, Martínez-Pastor JC, Pereira A, Marcos M, Soriano A. Length of storage of transfused red blood cells and risk of prosthetic joint infection after primary knee arthroplasty. J Arthroplasty 2014;29(10):2016-2020

17 Ballantyne A, Walmsley P, Brenkel I. Reduction of blood transfusion rates in unilateral total knee arthroplasty by the introduction of a simple blood transfusion protocol. Knee 2003;10 (04):379-384

18 Salido JA, Marín LA, Gómez LA, Zorrilla P, Martínez C. Preoperative hemoglobin levels and the need for transfusion after prosthetic hip and knee surgery: analysis of predictive factors. J Bone Joint Surg Am 2002;84(02):216-220

19 Guerin S, Collins C, Kapoor H, McClean I, Collins D. Blood transfusion requirement prediction in patients undergoing primary total hip and knee arthroplasty. Transfus Med 2007;17 (01):37-43

20 Noticewala MS, Nyce JD, Wang W, Geller JA, Macaulay W. Predicting need for allogeneic transfusion after total knee arthroplasty. J Arthroplasty 2012;27(06):961-967

21 Ogbemudia AE, Yee SY, MacPherson GJ, Manson LM, Breusch SJ. Preoperative predictors for allogenic blood transfusion in hip and knee arthroplasty for rheumatoid arthritis. Arch Orthop Trauma Surg 2013;133(09):1315-1320

22 Ahmed I, Chan JK, Jenkins P, Brenkel I, Walmsley P. Estimating the transfusion risk following total knee arthroplasty. Orthopedics 2012;35(10):e1465-e1471

23 Bong MR, Patel V, Chang E, Issack PS, Hebert R, Di Cesare PE. Risks associated with blood transfusion after total knee arthroplasty. J Arthroplasty 2004;19(03):281-287

24 Park JH, Rasouli MR, Mortazavi SM, Tokarski AT, Maltenfort MG, Parvizi J. Predictors of perioperative blood loss in total joint arthroplasty. J Bone Joint Surg Am 2013;95(19): 1777-1783 